\title{
Boundary conditions for the simulation of wave breaking
}

\author{
BENEDETTA IELE, FEDERICA PALLESCHI, FRANCESCO GALLERANO \\ Department of Civile, Constructional and Environmental Engineering \\ "Sapienza" University of Rome \\ Via Eudossiana 18, 00184 \\ ITALY
}

\begin{abstract}
In this paper we propose a new numerical model for the simulation of the wave breaking. The threedimensional equations of motion are expressed in integral contravariant form and are solved on a curvilinear boundary conforming grid that is able to represent the complex geometry of coastal regions. A time-dependent transformation of the vertical coordinate that is a function of the oscillation of the turbulent wave boundary layer is proposed. A new numerical scheme for the simulation of the resulting equations is proposed. New boundary conditions at the free surface and bottom for the equations of motion expressed in contravariant form are proposed. We present an analysis of the importance of the correct positioning, inside the oscillating turbulent boundary layer, of the centre of the calculation grid cell closest to the bottom, in order to correctly simulate the height of the breaking waves.
\end{abstract}

Key-Words: - Wave breaking, three-dimensional model, integral contravariant form, time-dependent curvilinear coordinate system, boundary conditions, moving grids in boundary layer.

Received: September 14, 2019. Revised: January 27, 2020. Re-revised: February 18, 2020.

Accepted: February 24, 2020. Published: February 28, 2020.

\section{Introduction}

In hydraulic engineering, the simulation of the hydrodynamic fields and turbulence under breaking waves allow the analysis of the effect produced by coastal defence structures and the modifications of the shoreline. One of the most used approaches for the simulation of breaking waves is based on the twodimensional depth-averaged equations of motion [14], that are obtained by assuming a simplified distribution of the hydrodynamic quantities along the vertical direction (depth averaged models).

In the literature, the three-dimensional simulation of wave induced free surface flows can carried out by numerical models that integrate the threedimensional Navier- Stokes equations, in which the so-called $\sigma$ transformation is used. In such a framework, the vertical Cartesian coordinate is transformed in a vertical coordinate that moves with the free surface. The adoption of shock-capturing numerical schemes in the $\sigma$-coordinate models allows the simulation of breaking waves. In these $\sigma$ coordinates shock-capturing models, the equations of motion are written in terms of Cartesian based conserved variables and are solved on a coordinate system that includes a time-varying vertical coordinate [5-6].

In a more recent class of numerical models, the three-dimensional equations of motion are expressed in a boundary conforming curvilinear coordinate system, where the vector and tensor quantities are expressed in a Cartesian frame of reference and only the vertical coordinate varies over time [7-8]. In these models, that aspire to use a computational grid with a reduced number of points along the vertical direction (of the order of a ten), the simulated wave height is in good agreement with respect to the experimental measures only before the wave breaking point. Ma et al., [5] demonstrated that, in general, the $\sigma$-coordinate shock-capturing models underestimate the wave breaking energy dissipation and overestimate the wave height in the surf-zone.

In this paper, we propose a time-dependent transformation of the vertical coordinate that is a function also of the thickness of the oscillating turbulent wave boundary layer.

To the knowledge of the writers, to date, there are no models in the literature, that solve the equations of motion on a computational grid with a reduced number of points along the vertical direction, that are able to simulate with a high level of accuracy the initial wave breaking point and the wave height in the surf-zone.

The lack of high level of accuracy, in the wave breaking simulation, depends not only on too dissipative turbulence models, but also on the errors produced by the numerical schemes. Furthermore, the above-mentioned high accuracy depends also on the way by which the boundary conditions at the bottom and free-surface are assigned in the momentum equation and on the way by which, in particular, the turbulence phenomena are represented along the water column. In the literature, in the way to assign the boundary conditions at the bottom there 
is a contradiction. As it is known, the turbulent boundary layer can be subdivided in three regions: the viscous sub-layer is the region closest to the bottom and is characterized by the dominance of the viscous stress; the buffer layer is the intermediate region and is characterized by the equal importance of viscous and turbulent stresses; the turbulent core is the region which is further from the bottom and where the turbulent stresses are dominant.

In the literature, the velocity boundary conditions and the friction velocity are deduced from appropriate logarithmic law.

In the calculation cell closest to the bottom, the cell-averaged velocity is obtained from the numerical solution of the momentum equation: this value is usually located in the centre of the above-mentioned grid cell. From this cell averaged velocity value, the friction velocity and the velocity boundary condition are obtained by the logarithmic law. The logarithmic law is valid in the turbulent core and, consequently, the centre of the calculation cell closest to the bottom (where the cell averaged velocity is calculated by the momentum equation) must necessarily be located inside of the turbulent core.

Placing the centre of the calculation cell closest to the bottom outside the boundary layer, or too close to the bottom (in the buffer layer or even in the viscous sub-layer), produces wrong evaluations of the friction velocity and wrong velocity boundary conditions and turbulent stresses in the turbulent boundary layer. In the current numerical models falling within the framework of the $\sigma$-coordinate transformation, the above-mentioned centre of the calculation cell closest to the bottom oscillates coherently with the oscillations of the free-surface, but in contradictory form with respect to the oscillations of the turbulent wave boundary layer: consequently, during the wave period, the centre of the calculation cell closest to the bottom is located outside or inside the turbulent wave boundary layer. It is evident that, in these numerical schemes, wrong evaluations of velocity boundary conditions, friction velocity and bottom stresses occur, with a consequent wrong simulation of the wave height at the breaking point and in the surf-zone.

In this work, a new model for the simulation of breaking waves is proposed. The three-dimensional equations of motion are expressed in integral contravariant form in time varying coordinates that can adapt to free-surface movements and to the evolution of the turbulent boundary layer thickness and are solved on boundary conforming curvilinear grids that can reproduce the complex geometry of the coastal regions.
In [9-10], the numerical scheme is based on a fractional-step method in which the sequence of steps to update the numerical solution consists in the calculation of a predictor velocity field, followed by a corrector step (in which a Poisson-like equation is numerically solved) and, finally, by the updating of the free-surface elevation. Furthermore, in [9-10] the finite difference numerical approximation of the differential terms in the Poisson-like equation are expressed in conservative form.

In the proposed numerical model, the integral contravariant form of the continuity and momentum equations are discretized by a finite-volume shockcapturing that uses an HLL approximate Riemann solver [11] and are updated by a fractional-step method that is different from the one presented in [910]. In the proposed fractional step method, the calculation of the predictor velocity field is followed by updating the free surface elevation and, finally, by a corrector step based on the numerical solution of a Poisson-like equation. Moreover, the differential terms in the Poison-like equation are expressed in non-conservative form in order to avoid the errors produced by the introduction of the metric terms in the finite difference approximations of the differential operators. New boundary conditions at the free surface and bottom for the contravariant equations are proposed. We present an analysis of the importance of the correct positioning, inside the oscillating turbulent boundary layer, of the centre of the calculation cell closest to the bottom, in order to correctly simulate the height of the breaking waves.

In this work, the centre of the calculation cell closest to the bottom oscillates coherently with the oscillations of the turbulent wave boundary layer and is always located in the turbulent core. The boundary condition for the cell averaged flow velocity, that is deduced form the logarithmic law, and for the eddy viscosity (which intervenes in the closure relation for the turbulent stress tensor in the turbulent boundary layer) are assigned on the lower face of the calculation cell closest to the bottom, that is to say in the lower part of the turbulent core, close to the buffer layer, where the balance between the production and dissipation of turbulent kinetic energy take place.

\section{Governing Equations}

In this paper we adopt the governing equations proposed in [9-10] in which the Navier-Stokes equations are expressed in integral contravariant form in a time-dependent curvilinear coordinate system. 


$$
\begin{gathered}
\frac{d}{d \tau} \int_{\Delta V_{0}}\left(\overrightarrow{\tilde{g}}^{(l)} \cdot \vec{g}_{(k)} \rho u^{k} \sqrt{g}\right) d \xi^{1} d \xi^{2} d \xi^{3}+ \\
\sum_{\alpha=1}^{3}\left\{\int_{\Delta A_{0}^{\alpha+}}\left(\overrightarrow{\tilde{g}}^{(l)} \cdot \vec{g}_{(k)} \rho u^{k}\left(u^{\alpha}-v^{\alpha}\right) \sqrt{g}\right) d \xi^{\beta} d \xi^{\gamma}-\right. \\
\left.\int_{\Delta A_{0}^{\alpha-}}\left(\overrightarrow{\tilde{g}}^{(l)} \cdot \vec{g}_{(k)} \rho u^{k}\left(u^{\alpha}-v^{\alpha}\right) \sqrt{g}\right) d \xi^{\beta} d \xi^{\gamma}\right\}= \\
\int_{\Delta V_{0}}\left(\overrightarrow{\tilde{g}}^{(l)} \cdot \vec{g}_{(k)} \rho f^{k} \sqrt{g}\right) d \xi^{1} d \xi^{2} d \xi^{3}+ \\
\sum_{\alpha=1}^{3}\left\{\int_{\Delta A_{0}^{\alpha+}}\left(\overrightarrow{\tilde{g}}^{(l)} \cdot \vec{g}_{(k)} R^{k \alpha} \sqrt{g}\right) d \xi^{\beta} d \xi^{\gamma}-\right. \\
\left.\int_{\Delta A_{0}^{\alpha-}}\left(\overrightarrow{\tilde{g}}^{(l)} \cdot \vec{g}_{(k)} R^{k \alpha} \sqrt{g}\right) d \xi^{\beta} d \xi^{\gamma}\right\} \\
\frac{d}{d \tau} \int_{\Delta V_{0}}(\rho \sqrt{g}) d \xi^{1} d \xi^{2} d \xi^{3}+ \\
\sum_{\alpha=1}^{3}\left\{\int_{\Delta A_{0}^{\alpha+}}\left(\rho\left(u^{\alpha}-v^{\alpha}\right) \sqrt{g}\right) d \xi^{\beta} d \xi^{\gamma}-\right. \\
\left.\int_{\Delta A_{0}^{\alpha-}}\left(\rho\left(u^{\alpha}-v^{\alpha}\right) \sqrt{g}\right) d \xi^{\beta} d \xi^{\gamma}\right\}=0
\end{gathered}
$$

where $u^{k}(k=1,3)$ is the contravariant component of the fluid velocity; $v^{\alpha}(\alpha=1,3)$ is the contravariant component of the velocity of the moving coordinate lines; $\rho$ is the water density; $f^{k}$ and $R^{k \alpha} \quad(k, \alpha=1,3)$ are, respectively, the contravariant component of the external body forces for unit mass vector and the contravariant components of the stress tensor. In the above equations $\tau$ is the time and $\xi^{1}, \xi^{2}, \xi^{3}$ are moving curvilinear coordinates obtained from the Cartesian coordinate system $\left(x^{1}, x^{2}, x^{3}, t\right)$ by a time-dependent transformation $x^{i}=x^{i}\left(\xi^{1}, \xi^{2}, \xi^{3}, \tau\right), t=\tau$. Vectors $\vec{g}_{(l)}$ and $\vec{g}^{(l)}$ are, respectively, the covariant and contravariant base vectors of the curvilinear coordinate system; $\sqrt{g}$ is the Jacobian of the transformation. $\Delta V_{0}=\Delta \xi^{1} \Delta \xi^{2} \Delta \xi^{3}$ is the volume element in the transformed space and $\Delta A_{0}^{\alpha+}$ and $\Delta A_{0}^{\alpha-}$ indicate the contour surfaces of the volume $\Delta V_{0}$ on which $\xi^{\alpha}$ is constant and which are located at the larger and at the smaller value of $\xi^{\alpha}$ respectively. Here, the indexes $\alpha, \beta$, and $\gamma$ are cyclic.

Equations (1) and (2) represent the general integral form of the Navier-Stokes equations expressed in a time dependent curvilinear coordinate system. The complete derivation of these equations can be found in [10]. In [9] it has been demonstrated that, by taking the limit as the volume approaches zero, the integral Equations (1) and (2) are reduced to the complete differential form of the contravariant Navier-Stokes equations in a time dependent curvilinear coordinate system that have been proposed in the literature by Luo and Bewley [12].

In this paper, in order to simulate the fully dispersive wave processes and the wave breaking, we start from the model proposed in [9-10] and obtain the following governing equations

$$
\begin{aligned}
& \frac{\partial \overline{H u^{l}}}{\partial \tau}=- \\
& \frac{1}{\Delta V_{0} \sqrt{g_{0}}} \sum_{\alpha=1}^{3}\left\{\int _ { \Delta A _ { o } ^ { \alpha + } } \left[\overrightarrow{\tilde{g}}^{(l)} \cdot \vec{g}_{(k)} H u^{k}\left(u^{\alpha}-v^{\alpha}\right)+\overrightarrow{\tilde{g}}^{(l)}\right.\right. \\
& \left.\cdot \vec{g}^{(\alpha)} G H^{2}\right] \sqrt{g_{0}} d \xi^{\beta} d \xi^{\gamma}- \\
& \int_{\Delta A_{o}^{\alpha-}}\left[\overrightarrow{\tilde{g}}^{(l)} \cdot \vec{g}_{(k)} H u^{k}\left(u^{\alpha}-v^{\alpha}\right)+\overrightarrow{\tilde{g}}^{(l)}\right. \\
& \left.\left.\cdot \vec{g}^{(\alpha)} G H^{2}\right] \sqrt{g_{0}} d \xi^{\beta} d \xi^{\gamma}\right\}+ \\
& \frac{1}{\Delta V_{0} \sqrt{g_{0}}} \sum_{\alpha=1}^{3}\left\{\int_{\Delta A_{o}^{\alpha+}} \overrightarrow{\tilde{g}}^{(l)} \cdot \vec{g}^{(\alpha)} G h H \sqrt{g_{0}} d \xi^{\beta} d \xi^{\gamma}-\right. \\
& \left.\int_{\Delta A_{o}^{\alpha-}} \overrightarrow{\tilde{g}}^{(l)} \cdot \vec{g}^{(\alpha)} G h H \sqrt{g_{0}} d \xi^{\beta} d \xi^{\gamma}\right\}+ \\
& \frac{1}{\Delta V_{0} \sqrt{g_{0}}} \sum_{\alpha=1}^{3}\left\{\int_{\Delta A_{0}^{\alpha+}} \overrightarrow{\tilde{g}}^{(l)} \cdot \vec{g}_{(k)} \frac{R^{k \alpha}}{\rho} H \sqrt{g_{0}} d \xi^{\beta} d \xi^{\gamma}-\right. \\
& \left.\int_{\Delta A_{o}^{\alpha-}} \overrightarrow{\tilde{g}}^{(l)} \cdot \vec{g}_{(k)} \frac{R^{k \alpha}}{\rho} H \sqrt{g_{0}} d \xi^{\beta} d \xi^{\gamma}\right\}- \\
& \frac{1}{\Delta V_{0} \sqrt{g_{0}}} \int_{\Delta V_{0}} \overrightarrow{\tilde{g}}^{(l)} \cdot \vec{g}^{(m)} \frac{\partial p_{d}}{\partial \xi^{m}} H \sqrt{g_{0}} d \xi^{1} d \xi^{2} d \xi^{3} \\
& \frac{\partial \bar{H}}{\partial \tau}=\frac{1}{\Delta A_{o}^{3} \sqrt{g_{0}}} \sum_{\alpha=1}^{2}\left[\int_{0}^{1} \int_{\Delta \xi_{o}^{\alpha+}} u^{\alpha} H \sqrt{g_{0}} d \xi^{\beta} d \xi^{3}\right. \\
& \left.-\int_{0}^{1} \int_{\Delta \xi_{o}^{\alpha-}} u^{\alpha} H \sqrt{g_{0}} d \xi^{\beta} d \xi^{3}\right]
\end{aligned}
$$

where $H=h+\eta$ is the total water depth; $h$ is the undisturbed water depth and $\eta$ is the free surface elevation with respect to the undisturbed water level; $G$ is the gravity acceleration; pressure $p$ is divided into a hydrostatic part, $\rho G\left(\eta-x^{3}\right)$ (the vertical coordinate $x^{3}$ is zero at the still free surface and it is positive upwards), and a dynamic one, $p_{d}$. The curvilinear coordinates $\xi^{1}, \xi^{2}, \xi^{3}, \tau$ are defined as

$$
\begin{gathered}
\xi^{1}=\xi^{1}\left(x^{1}, x^{2}, x^{3}\right) \quad ; \quad \xi^{2}=\xi^{2}\left(x^{1}, x^{2}, x^{3}\right) \quad ; \\
\xi^{3}=\frac{x^{3}+h\left(x^{1}, x^{2}\right)}{H\left(x^{1}, x^{2}, x^{3}, t\right)} \quad ; \quad \tau=t
\end{gathered}
$$

where $\xi^{1}$ and $\xi^{2}$ are the horizontal boundary conforming curvilinear coordinates and $\xi^{3}$ is the time 
varying vertical coordinate by which the irregular varying domain in the physical space is mapped into a regular fixed domain in the transformed space. $\sqrt{g}=\vec{k} \cdot\left|\vec{g}_{(1)} \wedge \vec{g}_{(2)}\right|$, where $\Lambda$ indicates the vector product. $\bar{H}$ and $\overline{H u^{l}}$ are spatial average values over volume elements defined in the form

$$
\begin{gathered}
\bar{H}=\frac{1}{\Delta A_{0}^{3} \sqrt{g_{0}}} \int_{\Delta A_{o}^{3}} H \sqrt{g_{0}} d \xi^{1} d \xi^{2} \\
\overline{H u^{l}}=\frac{1}{\Delta V_{0} \sqrt{g_{0}}} \int_{\Delta V_{0}} \overrightarrow{\tilde{g}}^{(l)} \cdot \vec{g}_{(k)} u^{k} H \sqrt{g_{0}} d \xi^{1} d \xi^{2} d \xi^{3}
\end{gathered}
$$

\section{Boundary conditions}

\subsection{Bottom boundary condition}

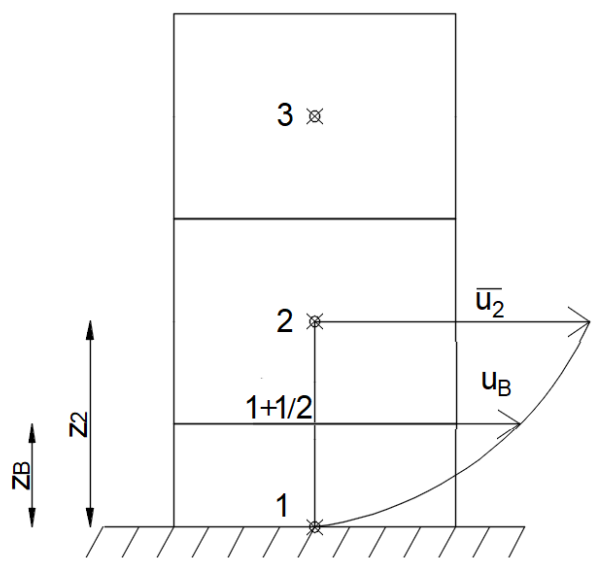

Fig. 1 Computational grid cells at the bottom

In Fig. 1 are shown the first grid cells near the bottom. For the sake of simplicity, let us consider flat bottom. Let $z_{2}$ be the distance from the bottom of the centre of the first calculation cell closest to the bottom (indicated whit 2 in Fig. 1); let $z_{B}$ be the distance from the bottom of the lower face of the first calculation cell closest to the bottom. The centre of the grid cell 1 belong to the bottom (as shown in Fig. 1 ). The cartesian based velocity cell averaged $\overline{u_{2}}$, indicated in Fig. 1, is deducted from the contravariant components of the velocity, obtained by Equations (3) and (4). This cell averaged velocity, $\overline{u_{2}}$, is placed at the centre of the cell 2 .

The logarithmic law used in order to calculate the friction velocity $u^{*}$ is

$$
\frac{\overline{u_{2}}}{u^{*}}=\frac{1}{\kappa} \ln \left(\frac{E u^{*} z_{2}}{v}\right)
$$

where $E=0.9$ is a coefficient used for a smooth wall; $\kappa=0.41$ is the von Kármán constant and $v$ is the kinematic viscosity. $u_{B}$ is the velocity boundary condition and it is calculated at the point $1+1 / 2$ (as shown in Fig. 1), on the lower face of the calculation cell closest to the bottom: this value is calculated using the logarithmic law $\left(u_{B}=\right.$ $\left.u^{*} / \kappa \ln \left(E u^{*} z_{B} / v\right)\right)$, through the friction velocity $u^{*}$.

As known, the turbulent boundary layer is divided in three regions: the viscous sub-layer is characterized only by the presence of the viscous stresses, $\left(y^{+} \leq 5\right.$ where $y^{+}=\frac{z u^{*}}{v}$ and $z$ is the distance from the wall in a Cartesian coordinate system); the buffer layer is characterized by the presence of the viscous stresses and turbulent stresses $\left(5<y^{+}<30\right)$; the turbulent core is characterized by the dominant presence of turbulent stresses $(30<$ $\left.y^{+}<100\right)$. The logarithmic law is valid in the turbulent core and consequently, the point at the centre of the calculation cell, in which the velocity is calculated, (in Fig. 1 is the point 2, distant $z_{B}$ from the wall) needs to be placed in the turbulent core.

In the $\sigma$-coordinate models present in the literature, the points position $1+1 / 2$ and 2 oscillates consistent whit the free surface movement, as shown in Fig. 2(a). Consequently, in the above-mentioned models the points $1+1 / 2$ and 2 , during the wave period, are located out the oscillating turbulent wave boundary layer, or in the turbulent core, or in the buffer layer, or in the viscous sub-layer. Hence, wrong values of the velocity boundary condition and the turbulent stress near the wall are produced. These wrong values influence the phase-averaged crest elevations.

In this paper, we propose an analysis on the importance of the correct placement of the points $1+$ $1 / 2$ and 2 in the turbulent core in order to correctly evaluate the phase-averaged crest elevations.

The turbulent closure relation used in this paper is expressed as follows

$$
R^{m n}=2 v^{\prime} S^{m n}
$$

where $R^{m n}$ are the contravariant components of the stress tensor; $v^{\prime}=v+v_{t}$ is the sum of the kinetic viscosity $v$ and the turbulent eddy viscosity $v_{t} ; S^{m n}$ are the contravariant components of strain rate tensor.

The turbulent stress boundary condition, $\tau^{m n}$, collocated on the lower face of the calculation cell closest to the bottom, is calculated using the turbulent eddy viscosity

$$
v_{t}=\kappa u^{*} z_{B}
$$

Outside the turbulent boundary layer, the turbulent eddy viscosity is evaluated by $v_{t}=$ $\left(C_{s} \Delta\right)^{2} \sqrt{2 S^{m n} S^{m n}}$, where $\Delta=\sqrt[3]{\Delta \xi^{1} \Delta \xi^{2} \Delta \xi^{3}}$ is the length scale dependent on the grid size and $C_{S}$ is the Smagorinsky coefficient. 
The Equation (9) is deducted by the hypothesis of the balance between the production and the dissipation of turbulent kinetic energy, that is holds true on the lower part of the turbulent core, near the buffer layer.

In this paper the points $1+1 / 2$ and 2 oscillate coherently with the turbulent boundary layer, as shown in Fig. 2(b). In particular, the point 2 is always located in the turbulent core; the point $1+1 / 2$ is also located in the turbulent core near the buffer layer, where is holds true the balance between the production and dissipation of turbulent kinetic energy. In Fig. 2(b) it is possible to notice that the first cell thickness increases in correspondence of the reduction of the velocity at the bottom and it reduces in correspondence of the increase of the velocity at the bottom.
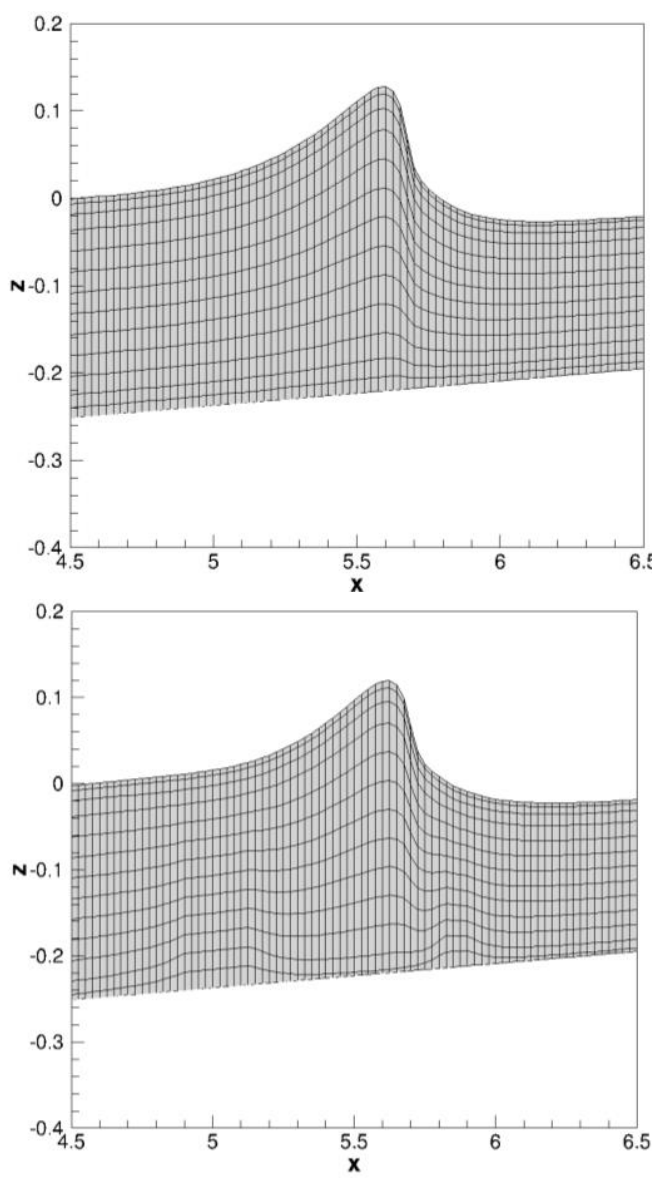

Fig. 2 Instantaneous representation of: a) computational grid in which the points $1+1 / 2$ and 2 move whit the free surface; b) computational grid in which the points $1+1 / 2$ and 2 move with the oscillating wave boundary layer.

\subsection{Surface boundary condition}

The boundary conditions for the dynamic pressure fields are derived from considering that, at the free- surface, the normal stresses are not equal to zero at all time.

$$
\begin{gathered}
R_{\text {ext }}^{33}=-\frac{p_{a} H}{\rho} \\
R_{\text {int }}^{33}=-\frac{p_{a} H}{\rho}-\frac{p_{d} H}{\rho}+R^{33}
\end{gathered}
$$

where $p_{a}$ is the atmospheric pressure; $R_{\text {ext }}^{33}$ and $R_{\text {int }}^{33}$ are, respectively, the normal external and internal stress tensor; $R^{33}$ is the normal stress tensor due to the turbulence and viscosity. The continuity of the normal stress reads

$$
R_{\text {int }}^{33}=R_{\text {ext }}^{33}
$$

Introducing Equations (10) and (11) in Equation (12) we obtain

$$
p_{d}=\frac{\rho}{H} R^{33}
$$

The normal stress tensor is expressed in contravariant formulation in a time-dependent curvilinear coordinates system as follows

$$
R^{33}=\frac{\vec{g}^{(3)}}{\left|\vec{g}^{(3)}\right|} \cdot \bar{R} \cdot \frac{\vec{g}^{(3)}}{\left|\vec{g}^{(3)}\right|}
$$

The components of the turbulent stress tensor $(\bar{R})$ are expressed as follow: $R^{m n}=2 v^{\prime} S^{m n}$, in which $S^{m n}$ the components of the strain rate are written as follow

$$
S^{m n}=\frac{1}{2} \sum_{i=1}^{3}\left[\frac{\partial\left(\vec{g}_{(j) m} u^{j}\right.}{\partial \xi^{(i)}} \vec{g}_{n}^{(i)}+\frac{\partial\left(\vec{g}_{(j) n} u^{j}\right.}{\partial \xi^{(i)}} \vec{g}_{m}^{(i)}\right]
$$

Introducing (15) in (13) we obtain the equation, written in contravariant formulation in a time-dependent curvilinear coordinate system, for the calculation of the dynamic pressure on the upper face of the top computational cell which correspond to the free surface.

$p_{d}=\frac{2 \rho v_{e f f}}{H}\left\{\frac{1}{2} \sum_{i=1}^{3}\left[\frac{\partial\left(\vec{g}_{(j) m} u^{j}\right.}{\partial \xi^{(i)}} \vec{g}_{n}^{(i)}+\frac{\partial\left(\vec{g}_{(j) n} u^{j}\right.}{\partial \xi^{(i)}} \vec{g}_{m}^{(i)}\right]\right\}$

\section{Numerical schemes}

The numerical scheme for the spatial discretization of the governing equations consists in a finite volume Shock capturing scheme in which TVD muscle reconstructions and the HLL approximate Riemann solver adopted in [9-10] are used to calculate the point values of water depth and flow velocity at the centre of the cell faces of the computational grid. Analogously to [9-10], the numerical procedure for 
updating the cell averaged 3D flow velocity field and free-surface elevation is based on a predictorcorrector method in conjunction with a two-stage second order Runge-Kutta method.

With respect to these papers, we propose two elements of originality: a modification of the procedure to update the free-surface elevation and a modification of the Poisson-like equation that characterize the corrector step. These two elements of originality entail a new procedure for the numerical solution of the flow velocity and freesurface elevation that can be summarized into the following steps:

1) Predictor step.

At each stage of the Runge-Kutta method, an approximate velocity field (called predictor velocity field) is carried out by numerically integrating the momentum equation, Equation (3), devoid of the dynamic pressure $\mathrm{q}$, by a shock-capturing numerical scheme in which MUSCLE-TVD reconstructions and the HLL approximate Riemann solver are used to obtain the point values of flow velocity and water depth at the centre of the computational cell faces.

2) Updating of the free surface elevation.

At the end of the predictor step, the point values of the water depth and flow velocity obtained at the centre of the cell faces by the local solution of the Riemann Problem are integrated over the vertical faces of the water column in order to calculate (by equation 4) the new position of the cell averaged freesurface elevation.

3) Corrector step.

The new position of the free-surface is used to recalculate the position of all the grid points and to update the metric terms that relate coordinates, vectors and tensors expressed in the irregular and time-varying physical domain occupied by the water to the ones expressed in a fixed regular computational domain obtained by the time dependent coordinate transformation; in the updated geometry, a Poissonlike equation is solved, by an iterative procedure, to calculate the scalar field $\psi$; the irrotational corrector velocity field is obtained as the gradient of the scalar field $\psi$ and is summed to the predictor one, in order to produce a final non-hydrostatic divergence-free velocity field.

Differently from what is done in [9-10], where the free-surface elevation was calculated at the end of the corrector step, in this paper we update the free surface elevation at the end of the predictor step. Consequently, the position of the free surface is updated by using the point values of the flow velocity and water depth at the centre of the cell faces that result from the local solution of the approximate Riemann Solver used in the predictor step. This improves the shock capturing properties of the numerical procedure and allows to better simulate steep wave fronts and the wave breaking.

Furthermore, in order to improve the accuracy of the numerical solution in highly distorted grids, we propose a curvilinear form of the Poisson-like equation that is different from the one used in [9-10]. The corrector velocity field is the gradient of a scalar field $\psi$, which is obtained by imposing that the divergence of the gradient of $\psi$ is equal and opposite to the divergence of the predictor velocity field. In curvilinear coordinates, both the divergence and gradient operators can be expressed in two different forms: the so called conservative form, in which the Jacobian $\sqrt{g}$ (and metric terms) of the coordinate transformation are written inside the spatial derivatives with respect to the curvilinear coordinates; and the so-called non conservative form, in which (by invoking a well-known metric identity) the Jacobian of the transformation is not explicitly present in the differential operator and the metric terms are written outside the spatial derivatives [13]. In the papers of [9-10], the divergence of the gradient of the scalar $\psi$ (in the corrector step) is written in conservative form and, consequently, the numerical approximation of the Poisson-like equation proposed in [9-10] includes the discretization of the spatial derivatives of the Jacobian of the transformation. This introduces approximation errors in the numerical scheme that can make the solution very sensitive to the deformation of the computational cells. In order to avoid this problem, in this paper we propose the following Poisson like equation for the corrector step in which both the divergence and gradient operators are written in non-conservative form

$$
\vec{g}^{(\alpha)} \cdot \frac{\partial\left(\frac{\vec{g}^{(j)} \partial \Psi}{\partial \xi^{j}}\right)}{\partial \xi^{\alpha}}=-\vec{g}^{(\alpha)} \cdot \frac{\partial v^{*}}{\partial \xi^{\alpha}}
$$

in which, differently from [9-10], the Jacobian of the transformation, $\sqrt{g}$, is not present at all.

By adopting a second order finite difference scheme for the spatial derivatives of the divergence operator, the proposed non-conservative form of the Poisson like equation (17) reads

$$
\begin{aligned}
& \frac{\vec{g}_{i, j, k+\frac{1}{2}}^{(1)}}{\Delta \xi_{i, j, k+\frac{1}{2}}^{1}} \cdot\left[\left(\vec{g}^{j} \frac{\partial \Psi}{\partial \xi^{j}}\right)_{i+\frac{1}{2^{\prime}}, k+\frac{1}{2}}-\left(\vec{g}^{j} \frac{\partial \Psi}{\partial \xi^{j}}\right)_{i-\frac{1}{2}, j, k+\frac{1}{2}}\right]+ \\
& \frac{\vec{g}_{i, j, k+\frac{1}{2}}^{(2)}}{\Delta \xi_{i, j, k+\frac{1}{2}}^{1}} \cdot\left[\left(\vec{g}^{j} \frac{\partial \Psi}{\partial \xi^{j}}\right)_{i, j+\frac{1}{2}, k+\frac{1}{2}}-\left(\vec{g}^{j} \frac{\partial \Psi}{\partial \xi^{j}}\right)_{i, j-\frac{1}{2}, k+\frac{1}{2}}\right]+
\end{aligned}
$$




$$
\begin{aligned}
& \frac{\vec{g}_{i, j, k+\frac{1}{2}}^{(3)}}{\Delta \xi_{i, j, k+\frac{1}{2}}^{1}} \cdot\left[\left(\vec{g}^{j} \frac{\partial \Psi}{\partial \xi^{j}}\right)_{i, j, k+1}-\left(\vec{g}^{j} \frac{\partial \Psi}{\partial \xi^{j}}\right)_{i, j, k}\right]=- \\
& \left\{\frac{\vec{g}_{i, j, k+\frac{1}{2}}^{(1)}}{\Delta \xi_{i, j, k+\frac{1}{2}}^{1}}\left[\vec{v}^{*}{ }_{i+\frac{1}{2}, j, k+\frac{1}{2}}-\vec{v}^{*}{ }_{i-\frac{1}{2}, j, k+\frac{1}{2}}\right]\right. \\
& +\frac{\vec{g}_{i, j, k+\frac{1}{2}}^{(2)}}{\Delta \xi_{i, j, k+\frac{1}{2}}^{1}}\left[\vec{v}^{*}{ }_{i, j+\frac{1}{2}, k+\frac{1}{2}}\right. \\
& \left.-\vec{v}^{*}{ }_{i, j-\frac{1}{2}, k+\frac{1}{2}}\right]+ \\
& \left.\frac{\vec{g}_{i, j, k+\frac{1}{2}}^{(3)}}{\Delta \xi_{i, j, k+\frac{1}{2}}^{1}}\left[\vec{v}^{*}{ }_{i, j, k+1}-\vec{v}^{*}{ }_{i, j, k}\right]\right\}
\end{aligned}
$$

By applying the same discretization scheme to the first order derivatives on the left-hand side of Equation 18, we obtain a set of algebraic equations that can be written in the form:

$$
\begin{gathered}
b_{1} \Psi_{i, j-1, k-1}^{(s)}+b_{2} \Psi_{i-1, j, k-1}^{(s)}+b_{3} \Psi_{i, j, k-1}^{(s)}+ \\
b_{4} \Psi_{i+1, j, k-1}^{(s)}+b_{5} \Psi_{i, j+1, k-1}^{(s)}+b_{6} \Psi_{i-1, j-1, k}^{(s)}+ \\
b_{7} \Psi_{i, j-1, k}^{(s)}+ \\
b_{8} \Psi_{i+1, j-1, k}^{(s)}+b_{9} \Psi_{i-1, j, k}^{(s)}+b_{10} \Psi_{i, j, k}^{(s)} \\
+b_{11} \Psi_{i+1, j, k}^{(s)}+b_{12} \Psi_{i-1, j+1, k}^{(s)} \\
+b_{13} \Psi_{i, j+1, k}^{(s)}+b_{14} \Psi_{i+1, j+1, k}^{(s)}+ \\
\\
b_{15}^{(s)} \Psi_{i, j-1, k+1}^{(s)}+b_{16} \Psi_{i-1, j, k+1}^{(s)}+b_{17} \Psi_{i, j, k+1}^{(s)} \\
+b_{18}^{(s)} \Psi_{i+1, j, k+1}^{(s)}+b_{19} \Psi_{i, j+1, k+1}^{(s)} \\
=R_{i, j, k+\frac{1}{2}}
\end{gathered}
$$

The $R_{i, j, k+\frac{1}{2}}$ represents the divergence (changed in sign) of the predictor velocity field in which $b_{l}$ are 19 coefficients.

As a consequence of the new non-conservative curvilinear form of the Poisson-like equation proposed in this paper, the $19 b_{l}$ coefficients of Equation 19 do not involve the Jacobian of the coordinate transformation $\sqrt{g}$. This choice produce a numerical solution that is less sensitive to the deformation of the computational cells in presence of distorted curvilinear grids, especially in the simulation of breaking waves.

The solution of the algebraic system defined by Equation (19) is carried out by an iterative multigrid technique in which the gauss-seidel lineby-line relaxation method is used (14).

\section{Rip current test}

In this Section, in order to validate and verify the ability of the new numerical procedure (PSC) and to underline the importance of the correct location of the calculation cell closest to the bottom at $y^{+}=40$, we numerically reproduce wave propagation, wave breaking and hydrodynamic velocity fields. To this end we reproduce a laboratory experiment carried out by Hamm [15]. The tank used by Hamm [15] measured $30 \mathrm{~m}$ by $30 \mathrm{~m}$; the sea bed consisted of a plane beach sloping at 1 in 30, with a rip channel excavated in the centre which produce a curved shaped coastline.

It is sufficient to reproduce only one-half of the basin because the basin is symmetric with respect to the y-axis. The turbulence stress tensor is estimated by the Smagorinsky sub-grid model in which the Smagorinsky coefficient is set to 0.21 . 

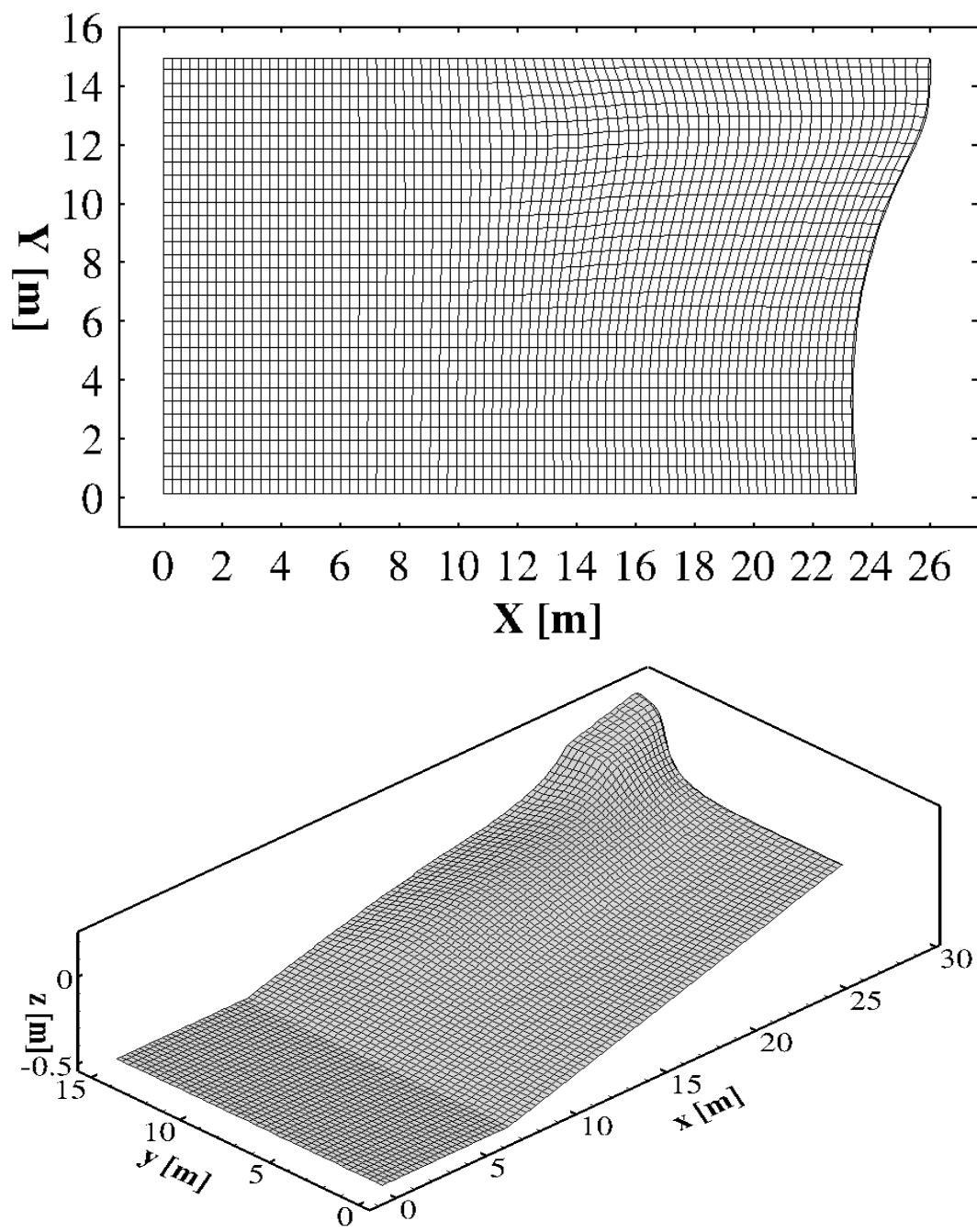

(a)

Fig. 3 a) Plan view of the curvilinear computational grid (Only one out of every five coordinate lines is shown). b): three-dimensional view of the bottom.

A plan view of the curvilinear computational grid and bottom variation, in which only one out of every five coordinate lines, are shown in Fig. 3. We numerically reproduce a regular wave train with period $T=1.25 \mathrm{~s}$ and height $H=0.07 \mathrm{~m}$.

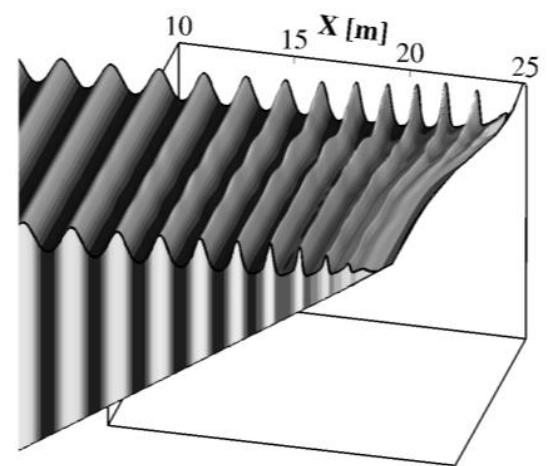

Fig. 4 Three-dimensional view detail of an instantaneous wave field at the time when the breaking induced circulation is fully developed.
A three-dimensional instantaneous wave field is shown in Fig. 14 where the nearshore currents are fully developed. Fig. 4 shows the wave height increase in correspondence with the channel location due to occurrence of a pronounced rip current along the channel.

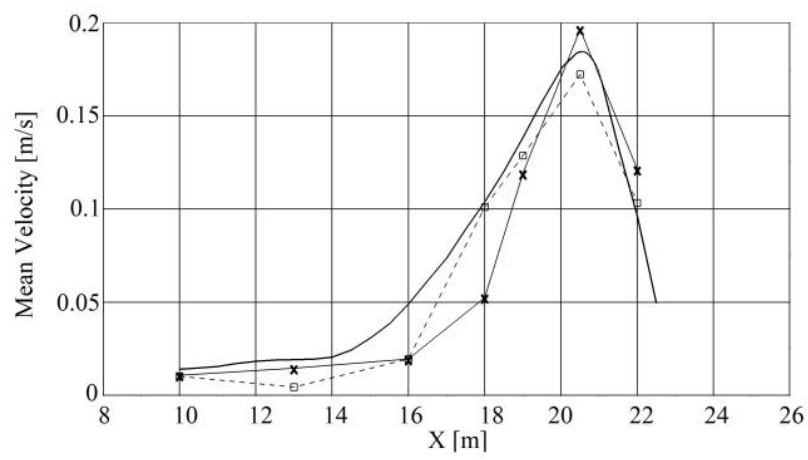

Fig. 5 Mean current velocity along the rip channel. Comparison between the experimental 
measurements [15] for unidirectional (crosses) and multidirectional (square) random waves and the numerical results (solid line).

Fig. 5 shows the time-average of the crossshore velocity components calculated near the bottom along the rip channel. From this Fig. it is possible to notice the good agreement between numerical result and experimental measurements [5].

\section{Results}

In this Section, the results of the numerical simulations of the wave breaking, obtained by the proposed model, are presented and are compared against the experimental data conducted by Ting and Kirby [16].

The experimental arrangement adopted by [16] consists in a sea bed with a sloping beach with slope 1:35. The still water depth is $h=0.4 m$. See Fig. 6 for a schematic view.

For the numerical simulations, the computational grid consists in: 13,728 grid cells in the horizontal direction with spacing $\Delta x_{1}=$ $0.025 \mathrm{~m} ; 13$ grid cells in the vertical direction. A cnoidal wave with period $T=2 s$ and wave height $H=0.125 m$, is imposed as input boundary condition.

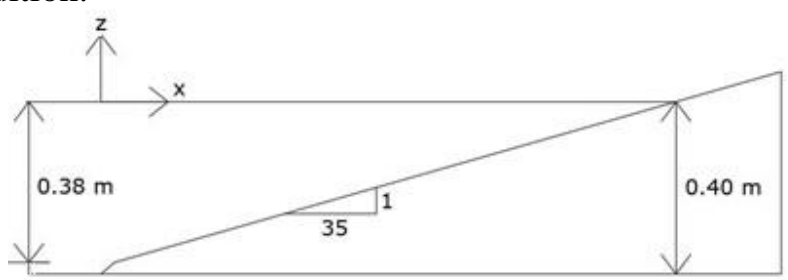

Fig. 6 Schematic experimental arrangement by Ting and Kirby [16]

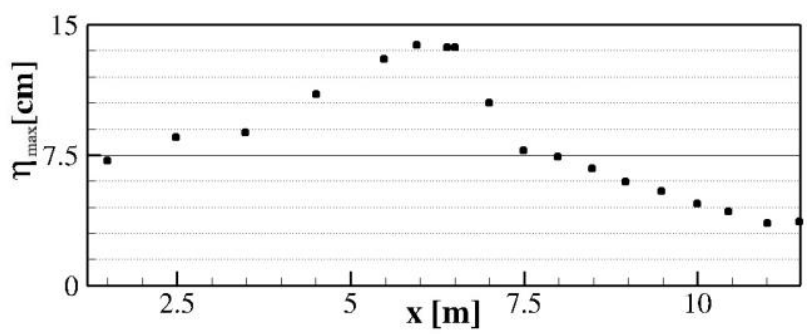

Fig. 7 Ting and Kirby [16] breaking wave test case. Phase-averaged crest elevations.

In Fig. 7 the experimental measurements by Ting and Kirby [16] in terms of the cross-shore distribution of crest, are shown.

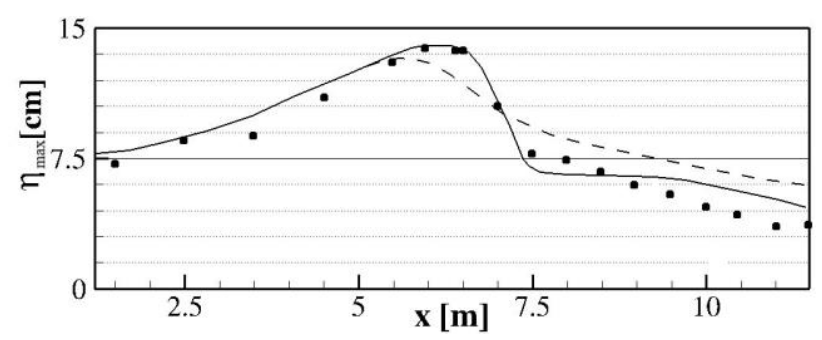

Fig. 8 Ting and Kirby [16] breaking wave test case. Phase-averaged crest elevations. Experimental data (circles) and numerical results obtained by PSC (solid line) and PCS (dashed line).

In Fig. 8 the comparison between the results obtained by using the numerical procedure of [9-10] and the results obtained by the numerical procedure proposed in this paper. The dashed line shows the cross-shore distribution of crest, obtained by the numerical procedure proposed in [9-10], which consists in a fractional-step method with the following sequence: evaluation of the predictor velocity field; evaluation of a corrector velocity field; evaluation of the free-surface elevation. Is to be noted that in the procedure PCS proposed in [9-10], the finite approximations of the differential terms that are present in the Poisson-like equation, are expressed in conservative form.

The solid line shows the cross-shore distribution of crest, obtained by the numerical procedure proposed in this paper, which consists in a fractional-step method with the following sequence: evaluation of the predictor velocity field; evaluation of the free-surface elevation; evaluation of a corrector velocity field. Is to be noted that in the procedure proposed in this paper (PSC), in order to avoid the errors produced by the introduction of the metric terms in the finite approximations of the differential operators that are present in the Poissonlike equation, these finite approximations are expressed in non-conservative form.

In both the numerical simulations, the boundary conditions proposed in this work are used and a Smagorisnky coefficient $C_{s}=0.21$ is adopted in the turbulent closure relation outside the turbulent boundary layer.

The distance between the wall and the lower face of the calculation cell closest to the bottom (in which the boundary conditions for the velocity and for the turbulent stress tensor, are imposed), oscillates coherently with respect to the turbulent boundary layer and it is set to the dimensionless wall distance $y^{+}=40$.

The numerical results obtained by means of the procedure PCS (dashed line in Fig. 8) show that the initial wave breaking point is located at $x=5.5 \mathrm{~m}$, 
much before the predicted location by the experimental results $(x=6.4 m)$; after the wave breaking, the cross-shore distribution of crest is underestimated until $x=7.0 \mathrm{~m}$ and their slope is lower than the experimentally predicted one; after $x=7.0 \mathrm{~m}$, the cross-shore distribution of crest is overestimated.

The numerical results obtained by means of the procedure PSC (solid line in Fig. 8) show that the initial wave breaking point $(x=6.4 m)$ and the slope of the cross-shore distribution of crest are well predicted until $x=7.25 \mathrm{~m}$; from $x=7.25 \mathrm{~m}$ to $x=$ $8.5 \mathrm{~m}$, the cross-shore distribution of crest is slightly underestimated; after $x=8.5 \mathrm{~m}$, the cross-shore distribution of crest is slightly overestimated.

From the comparison between the results obtained by the procedure PCS and the results obtained by the procedure PSC, it can be seen that the results obtained by the procedure PSC, in terms of crossshore distribution of crest, are in good agreement with the experimental measurements, in contrast to the results obtained by the procedure PCS [9-10].

The procedure PCS induces a high numerical dissipation, which causes the anticipation of the initial wave breaking point, the underestimation in the cross-shore distribution of crest and in their slope, and from a certain point onwards an overestimation of the cross-shore distribution of crest.

The procedure PSC proposed in this paper, allows us to better simulate the cross-shore distribution of crest and the initial wave breaking point, with respect to the procedure PCS. The procedure PSC induces a lower numerical dissipation than the procedure PCS, thanks to the fact that, with the non-conservative form of the Poisson-like equation, the errors produced by the introduction of the metric terms into the finite approximations of the differential operators, are avoided.

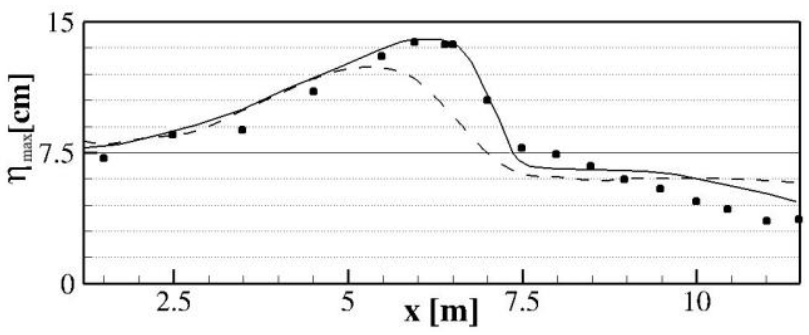

Fig. 9 Ting and Kirby [16] breaking wave test case. Phase-averaged crest elevations. Experimental data (circles) and numerical results obtained by $y^{+}$ average fixed value of 40 (solid line) and $y^{+}$fixed value of 40 (dashed line).
In Fig. 9, the comparison between the results obtained with an oscillation of the lower face of the calculation cell closest to the bottom, with the freesurface movement (coherently with $\sigma$-coordinate models), and the results obtained with an oscillation of the aforementioned face, with the turbulent boundary layer, is shown.

The dashed line shows the cross-shore distribution of crest obtained by the numerical simulation in which the distance between the wall and the lower face of the calculation cell closest to the bottom oscillates with the free-surface movement and it is set to a dimensionless wall distance that has a time-averaged value equal to 40 .

The solid line shows the cross-shore distribution of crest obtained by the numerical simulation in which the distance between the wall and the lower face of the calculation cell closest to the bottom coherently oscillates with the turbulent boundary layer and it is always set to a dimensionless wall distance $y^{+}=40$, and it is always located inside the turbulent core, near the buffer layer, where the hypothesis of balance between production and dissipation of turbulent kinetic energy holds true.

In both the numerical simulations, in the turbulent closure relation, outside the turbulent boundary layer, a Smagorisnky coefficient $C_{S}=$ 0.21 is adopted.

The numerical results obtained with an oscillation of the lower face of the calculation cell closest to the bottom, with the free-surface movement (dashed line in Fig. 9), show that the initial wave breaking point is located at $x=6.0 \mathrm{~m}$, much before the predicted location by the experimental results $(x=6.4 \mathrm{~m})$; from $x=4.8 \mathrm{~m}$ to $x=10.0 \mathrm{~m}$, the cross-shore distribution of crest is underestimated; after $x=10.0 \mathrm{~m}$, the cross-shore distribution of crest is overestimated.

The numerical results obtained with an oscillation of the lower face of the calculation cell closest to the bottom, with the turbulent boundary layer (solid line in Fig. 9) are in good agreement with the experimental measurement, as shown before, in the discussion of the Fig. 8.

The comparison between the results obtained with an oscillation of the lower face of the calculation cell closest to the bottom, with the free-surface movement, and the results obtained with an oscillation of the aforementioned face with the turbulent boundary layer, shows the limitation of the $\sigma$-coordinate models that are present in the literature.

In the $\sigma$-coordinate models, the lower face of the calculation cell closest to the bottom follows the free-surface movement, in a contradictory oscillation with respect to the one of the turbulent boundary 
layer. Consequently, in the $\sigma$-coordinate models, the lower face of the calculation cell closest to the bottom, during the wave period, may be located alternatively outside the turbulent boundary layer, inside the turbulent core, inside the buffer layer, or (when the velocity is low) inside the viscous sublayer. It is evident that in the aforementioned model, there is an erroneous evaluation of the velocity boundary condition, of the friction velocity and of the turbulent bottom stresses, which causes an erroneous simulation of the cross-shore distribution of crest.

In the scheme proposed in this work, the cellaveraged velocity value is located at the centre of the calculation cell closest to the bottom near the bottom; by means of this cell-averaged velocity value, through the logarithmic law defined in Section 4, the friction velocity $u^{*}$ and the velocity value at the lower face of the grid cell $u_{B}$, are computed.

The adopted logarithmic law holds true in the turbulent core. The relation used to calculate the turbulent eddy viscosity in the turbulent boundary layer is valid inside the turbulent core, near the buffer layer. The accordance between the numerical results and the experimental measurements, in terms of cross-shore distribution of crest and of location of the initial wave breaking point, demonstrates that it is necessary that the centre of the aforementioned grid cell oscillates coherently with the turbulent boundary layer and that the lower face of the aforementioned grid cell is placed near the buffer layer, where the balance between production and dissipation of turbulent kinetic energy holds true.

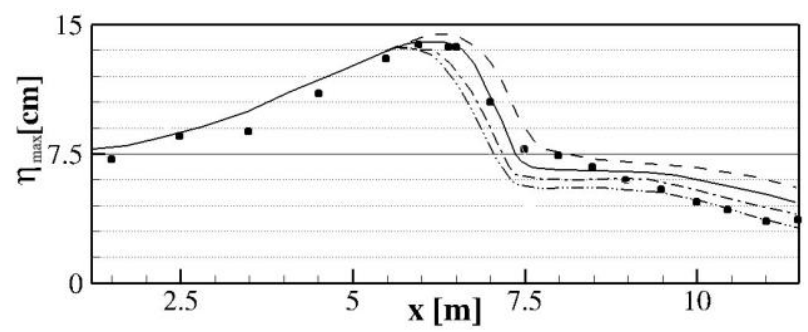

Fig. 10 Ting and Kirby [16] breaking wave test case. Phase-averaged crest elevation. Experimental data (circles) and numerical results with $y^{+}=80$ (dash dot dot line), $y^{+}=60$ (dash dot line), $y^{+}=$ 40 (solid line) and $y^{+}=30$ (dashed line).

In Fig. 10, the comparison among the results obtained with four numerical simulations, in which the lower face of the calculation cell closest to the bottom oscillates with the turbulent boundary layer, is shown. In Fig. 10, the cross-shore distribution of crest, obtained by the aforementioned four different simulations in which the distance between the wall and the lower face of the calculation cell closest to the bottom is set to a dimensionless wall distance $y^{+}=80$ (dash dot dot line), $y^{+}=60$ (dash dot line), $y^{+}=40$ (solid line) and $y^{+}=30$ (dotted line), is shown.

In all the numerical simulations, the boundary conditions and the procedure PSC proposed in this work, are adopted and, in the turbulent closure relation, outside the turbulent boundary layer, the Smagorisnky coefficient is set to $C_{s}=0.21$.

The numerical results obtained with the numerical simulation carried out with $y^{+}=80$ (dash dot dot line), show that the initial wave breaking point is located at $x=5.75 \mathrm{~m}$, much before the predicted location by the experimental results $(x=$ $6.4 \mathrm{~m}$ ) ; from $x=5.75 \mathrm{~m}$ to $x=9.5 \mathrm{~m}$, the crossshore distribution of crest is underestimated and their slope is lower than the one obtained by the experimental measurements.

The numerical results obtained with the numerical simulation carried out with $y^{+}=60$ (dash dot line), show that the initial wave breaking point is located at $x=6.25 \mathrm{~m}$, before the predicted location by the experimental results $(x=6.4 m)$; from $x=$ $6.25 \mathrm{~m}$ to $x=9.0 \mathrm{~m}$, the cross-shore distribution of crest is underestimated and their slope is comparable to the one obtained by the experimental measurements; after $x=9.0 \mathrm{~m}$, the cross-shore distribution of crest is slightly overestimated.

The numerical results obtained with the numerical simulation carried out with $y^{+}=40$ (solid line), have been already described and show the best agreement with the experimental results.

The numerical results obtained with the numerical simulation carried out with $y^{+}=30$ (dashed line), show that the initial wave breaking point is located at $x=6.5 \mathrm{~m}$, slightly after the predicted location by the experimental results $(x=$ $6.4 \mathrm{~m}$ ); after $x=6.5 \mathrm{~m}$, the cross-shore distribution of crest is overestimated and their slope is higher than the one obtained by the experimental measurements.

From the comparison among the different simulations carried out, it is possible to notice the importance to correctly locate the lower face of the calculation cell closest to the bottom. With the increase of the distance $z_{B}$ between the wall and the lower face of the calculation cell closest to the bottom: the friction velocity $u^{*}$ decreases; the velocity boundary condition $u_{B}$ decreases; the bottom turbulent stresses decrease, causing a decrease in the value of the cross-shore distribution of crest and an anticipation of the initial wave breaking point.

As stated before, the closure relation adopted for the evaluation of the turbulent eddy viscosity $v_{t}$ 
in the turbulent boundary layer, is deducted from the hypothesis of balance between production and dissipation of turbulent kinetic energy (which holds true inside the turbulent core, near the buffer layer).

The accordance of the numerical results (solid line in Fig. 9) with the experimental measurements, in terms of cross-shore distribution of crest and of location of the initial wave breaking point, demonstrates that it is necessary that the lower face of the calculation cell closest to the bottom, where the turbulent eddy viscosity $v_{t}$ is evaluated, oscillates at a dimensionless wall distance $y^{+}$of about 40 , inside the turbulent core near the buffer layer, where the balance between production and dissipation of turbulent kinetic energy holds true.

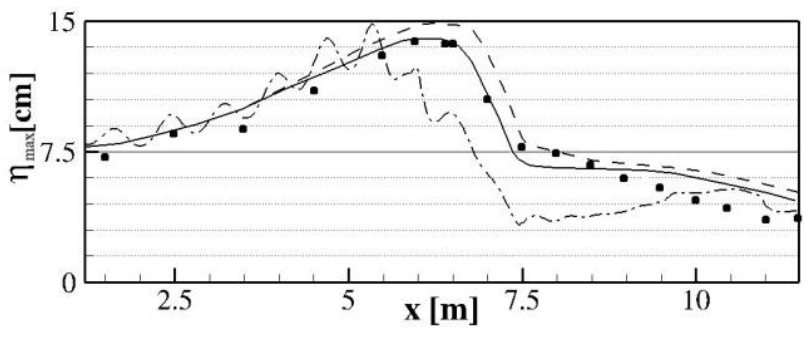

Fig. 11 Ting and Kirby [16] breaking wave test case. Phase-averaged crest elevation. Experimental data (circles) and numerical results with $C_{s}=0.14$ (dash dot line), $C_{s}=0.21$ (solid line) and $C_{s}=0.28$ (dashed line).

In Fig. 11, the comparison among the results obtained with three different numerical simulations in which in the turbulent closure relation, outside the turbulent boundary layer, the Smagorisnky coefficient is set to $C_{s}=0.14$ (dash dot line), 0.21 (solid line) and 0.28 (dashed line).

In Fig. 11, the cross-shore distribution of crest, obtained by means of the aforementioned three different simulations in which the distance between the wall and the lower face of the calculation cell closest to the bottom is set to a dimensionless wall distance $y^{+}=40$, is shown

The numerical simulation carried out with $C_{S}=0.14$ (dash dot line) is unstable, because of the low turbulent kinetic energy dissipation.

The results obtained by means of the numerical simulation carried out with $C_{s}=0.21$ (solid line), have been previously described in the discussion of Fig. 8 and show the best agreement with the experimental results.

The results obtained by means of the numerical simulation carried out with $C_{s}=0.28$ (dashed line), show that the initial wave breaking point is located at $x=6.85 \mathrm{~m}$, after the predicted location by the experimental results $(x=6.4 \mathrm{~m})$; after $x=4.25 \mathrm{~m}$, the cross-shore distribution of crest is overestimated.

From the comparison among the different simulations, carried out with different Smagorisnky coefficients, it is possible to notice that by adopting low $C_{S}$ in the turbulent closure relation outside the turbulent boundary layer, the numerical simulations are unstable, because of the low turbulent kinetic energy dissipation. High Smagorisnky coefficients cause an increase of the turbulent stresses, with a consequent overestimation of the cross-shore distribution of crest and erroneous prediction of the location of the initial wave breaking point.

\section{Conclusion}

In this paper we propose a new numerical model for the simulation of the wave breaking. The threedimensional equations are expressed in contravariant form and are solved over a curvilinear boundary conforming grid, which is capable to represent the complex geometries, typically present in coastal regions. A transformation over the time of the vertical coordinate, as a function of the movement of the oscillating turbulent boundary layer, other than the free-surface elevation. It has been demonstrated that the proposed numerical procedure allows us to correctly simulate the cross-shore distribution of crest and the location of the initial wave breaking point. It has been demonstrated that the centre of the first calculation cell must oscillate coherently with the turbulent boundary layer. It has been demonstrated that the lower face of the calculation grid cell, where the turbulent eddy viscosity $v_{t}$ is evaluated, must always oscillate inside the turbulent core and near the buffer layer, where the hypothesis of balance between production and dissipation of turbulent kinetic energy holds true.

\section{References:}

[1] Cannata G., Barsi L., Petrelli C., Gallerano F., Numerical investigation of wave fields and currents in a coastal engineering case study, WSEAS Transactions on Fluid Mechanics, Vol. 13, 2018, pp. 87-94.

[2] Shi F., Kirby J.T., Harris J.C., Geiman J.D., Grilli S.T., A high-order adaptive time-stepping TVD solver for Boussinesq modelling of breaking waves and coastal inundation. Ocean Model. 2012, 43-44, 36-51.

[3] Cannata G., Petrelli C., Barsi L., Fratello F., Gallerano F., A dam-break flood simulation model in curvilinear coordinates, WSEAS Transactions on Fluid Mechanics, Vol. 13, 2018, pp. 60-70. 
[4] Caleffi V., Valiani A., Li G., A comparison between bottom-discontinuity numerical treatments in the DG framework, Applied Mathematical Modelling, Vol. 40, No. 17-18, 2016, pp. 7516-7531.

[5] Ma G., Shi F., Kirby J.T., Shock-capturing nonhydrostatic model for fully dispersive surface wave processes, Ocean Modelling, Vol. 43-44, 2012, pp. 22-35.

[6] Bradford S.F., Non-hydrostatic model for surfzone simulation, Journal of Waterway, Port, Coastal, and Ocean Engineering, Vol. 137, No. 4, 2011, pp. 163-174.

[7] Cannata G., Petrelli C., Barsi L., Camilli F., Gallerano F., 3D free surface flow simulations based on the integral form of the equations of motion, WSEAS Transactions on Fluid Mechanics, Vol. 12, 2017, pp. 166-175.

[8] Cannata G., Gallerano F., Palleschi F., Petrelli C., Barsi L., Three-dimensional numerical simulation of the velocity fields induced by submerged breakwaters, International Journal of Mechanics, Vol. 13, 2019, pp. 1-14.

[9] Palleschi, F., Iele, B., Gallerano, F. Integral contravariant form of the Navier-Stokes equations. WSEAS Transactions on Fluid Mechanics, Vol. 14, 2019, pp. 101-113.

[10] Cannata G., Petrelli C., Barsi L., Gallerano F., Numerical integration of the contravariant integral form of the Navier-Stokes equations in time-dependent curvilinear coordinate systems for three-dimensional free surface flows, Continuum Mechanics and Thermodynamics, Vol. 31, No. 2, 2019, pp.491-519.

[11] Harten A., Lax P.D., vanLeer B., On upstream differencing and Godunov-Type Schemes for Hyperbolic Conservation Laws, SIAM Review, Vol.25, No. 1, 1983, pp. 35-61.

[12] Luo H., and Bewley T. R., On the contravariant form of the Navier-Stokes equations in time dependent curvilinear coordinate systems, Journal of Computational Physics, Vol. 199, No. 1, 2004, pp. 355-375.

[13] Thompson Joe F., Warsi Z.U.A., Wayne Mastin C., Numerical Grid Generation, North-Holland, 1985.

[14] Rosenfeld M., Kwak D., Time-dependent solutions of viscous incompressible flows in moving co-ordinates, International Journal of Numerical Methods in Fluids, Vol. 13, 1991, 1311-1328.

[15] Hamm L., Directional nearshore wave propagation over a rip channel: an experiment. Proceedings of the 23rd International
Conference of Coastal Engineering, 1992, pp. 226-239.

[16] Ting F.C.K., Kirby J.T., Observation of undertow and turbulence in a wave period, Costal Engineering, No.24, 1994, pp. 51-80. 